\title{
National Aeronautics and Space Administration (NASA) Environmental Control and Life Support (ECLS) Integrated Roadmap Development
}

\author{
Jordan Metcalf $^{1}$ and Laurie Peterson ${ }^{2}$ \\ NASA Johnson Space Center, Houston, Texas, 77062, USA \\ Robyn Carrasquillo ${ }^{3}$ and Robert Bagdigian ${ }^{4}$ \\ NASA Marshall Space Flight Center, Huntsville, Alabama, 35808
}

\begin{abstract}
At present, NASA has considered a number of future human space exploration mission concepts . Yet, detailed mission requirements and vehicle architectures remain mostly undefined, making technology investment strategies difficult to develop and sustain without a top-level roadmap to serve as a guide. This paper documents a roadmap for development of Environmental Control and Life Support Systems (ECLSS) capabilities required to enhance the long-term operation of the International Space Station (ISS) as well as enable beyond-Low Earth Orbit (LEO) human exploration missions. Three generic mission types were defined to serve as a basis for developing a prioritized list of needed capabilities and technologies. Those are 1) a short duration micro gravity mission; 2) a long duration transit microgravity mission; and 3) a long duration surface exploration mission. To organize the effort, ECLSS was categorized into three major functional groups (atmosphere, water, and solid waste management) with each broken down into sub-functions. The ability of existing state-of-the-art (SOA) technologies to meet the functional needs of each of the three mission types was then assessed by NASA subject matter experts. When SOA capabilities were deemed to fall short of meeting the needs of one or more mission types, those "gaps" were prioritized in terms of whether or not the corresponding capabilities enable or enhance each of the mission types. The result was a list of enabling and enhancing capabilities needs that can be used to guide future ECLSS development, as well as a list of existing hardware that is "ready to go" for exploration-class missions. A strategy to fulfill those needs over time was then developed in the form of a roadmap. Through execution of this roadmap, the hardware and technologies intended to meet exploration needs will, in many cases, directly benefit the ISS operational capability, benefit the Multi-Purpose Crew Vehicle (MPCV), and guide long-term technology investments for longer duration missions The final product of this paper is an agreed-to ECLSS roadmap detailing ground and flight testing to support the three mission scenarios previously mentioned. This information will also be used to develop the integrated NASA budget submit in January 2012.
\end{abstract}

\footnotetext{
${ }^{1}$ Insert Job Title, Department Name, Address/Mail Stop, and AIAA Member Grade for first author.
} 